\title{
Electron transport in a quasi-one dimensional channel on suspended helium films
}

\author{
Sviatoslav S. Sokolov \\ Departamento de Física, Universidade Federal de São \\ Carlos, 13565-905, São Carlos, São Paulo, Brazil and B. I. Verkin \\ Institute for Low Temperature Physics and Engineering, National Academy of \\ Sciences of Ukraine, 61103, Kharkov, Ukraine \\ Nelson Studart \\ Departamento de Física, Universidade Federal de São \\ Carlos, 13565-905, São Carlos, São Paulo, Brazil
}

(November 1, 2018)

\begin{abstract}
Quasi-one dimensional electron systems have been created using a suspended helium film on a structured substrate. The electron mobility along the channel is calculated by taking into account the essential scattering processes of electrons by helium atoms in the vapor phase, ripplons, and surface defects of the film substrate. It is shown that the last scattering mechanism may dominate the electron mobility in the low temperature limit changing drastically the temperature dependence of the mobility in comparison with that controlled by the electron-ripplon scattering.
\end{abstract}


There has been great interest in the study of the quasi-one-dimensional electron system (Q1DES) produced on the liquid helium surface [1-9] in which the motion of usual quasitwo-dimensional (Q2D) surface electrons (SE) [10] is restricted in one more spatial direction. In most methods to realize the Q1D electron system, the charged channels are formed when parallel strips of a dielectric substrate are filled with liquid helium due to the capillary forces. The curvature radius $R$ of the liquid into the strip can be varied in wide range. The profile of the liquid surface across the channel ( $y$ direction) can be assumed to have a semicircular form $z_{0}=R\left[1-\sqrt{1-(y / R)^{2}}\right] \simeq y^{2} / 2 R$ for $y \ll R$, where $R=\alpha / \rho g H$ with $\alpha$ and $\rho$ are the surface tension and the helium density, respectively, $g$ is the acceleration of the gravity, and $H$ is the bulk helium level below the stripped structure. The electron confinement across the channel is achieved by the action of a holding electric field $E_{\perp}$ along the normal direction to the liquid surface ( $z$ axis). As a result the electron near the channel bottom is subjected to the parabolic potential $U(y)=e E_{\perp} z_{0} \simeq m \omega_{0}^{2} y^{2} / 2$, where $\omega_{0}=\left(e E_{\perp} / m R\right)^{1 / 2}$. [11]

Quite recently, it was shown that very stable suspended helium films over a structured substrate can be created with arbitrary thickness, as depicted in Fig. 1. [0,12] Under certain conditions (the distance between the elevations must be smaller than the capillary length), the helium film does not follow the substrate form but fills the depressions through capillary condensation. The film thickness $d$ is controlled by channel width $W$ and the bulk level height $H$. This method is useful to laterally confine the electrons in a sub-micrometer case and generate Q1DES above helium films. This system can be modelled in a similar form as before, but an effective holding field $E_{\perp}^{*}$ replaces now $E_{\perp}$ in the confined frequency $\omega_{0}$ due to the contribution of the polarization interaction of the electron with the solid substrate: $E_{\perp}^{*}=E_{\perp}+\left(\Lambda_{1} / e\right)\left\langle 1\left|(z+d)^{-2}\right| 1\right\rangle=E_{\perp}+\left(2 \Lambda_{1} \gamma^{2} / e\right) f(\gamma d)$ where the angular brackets denote averaging over the electron wave function $|1\rangle=2 \gamma^{3 / 2} z \exp (-\gamma z)$ for the motion in the $z$ direction ( $\gamma$ is the localization parameter [13]), $\Lambda_{1}=e^{2} \varepsilon_{\mathrm{He}}\left(\varepsilon_{s}-\varepsilon_{\mathrm{He}}\right) /\left[\left(\varepsilon_{\mathrm{He}}+1\right)^{2}\left(\varepsilon_{s}+\varepsilon_{\mathrm{He}}\right)\right]$ with $\varepsilon_{\mathrm{He}}$ and $\varepsilon_{s}$ the dielectric constants of liquid helium and substrate, respectively; $d$ is film thickness near $y=0, f(x)=[1+2 x+4 x(1+x) \exp (2 x) \operatorname{Ei}(-2 x)]$, and $\operatorname{Ei}(x)$ is integral 
exponential function. According to our estimates polarization effects becomes dominant for $d \lesssim 10^{-6} \mathrm{~cm}$, especially for substrates with large $\varepsilon_{s}$.

For wide enough rectangular strips, the curvature effects can be discarded especially for small $d$ and high electron densities which lead to a flat profile in the centre of channel. However, charged electrodes may be arranged in such a way that the applied gate voltage produces an effective lateral electrostatic confinement. [6,9] The electrostatic potential should be calculated using the Poisson equation with appropriate boundary conditions. However for $|y| \ll W$, the same model can be used, but $U(y)$ now depends on $\omega_{\text {conf }}^{2}=\omega_{0}^{2}+\omega_{\text {es }}^{2}$, where $\omega_{\mathrm{es}}$ is the characteristic electrostatic frequency.

In this paper, we study the transport properties of the Q1DES over helium film. Despite of different possible ways to create the system, the electron states inside the channel can be simply described. Indeed the electron potential energy can be written as

$$
V(y, z)=e E_{\perp} z-\frac{\Lambda_{0}}{z}-\frac{\Lambda_{1}}{z+d}+\frac{m \omega_{\mathrm{conf}}^{2} y^{2}}{2}
$$

where $\Lambda_{0}=e^{2}\left(\varepsilon_{\mathrm{He}}-1\right)\left[4\left(\varepsilon_{\mathrm{He}}+1\right)\right]$ and the characteristics of the channel geometry are given by $\omega_{\text {conf }}$ within the harmonic approximation. The energy spectrum and the wave function for electron in the plane are $E_{n, k_{x}}=\hbar^{2} k_{x}^{2} / 2 m+\hbar \omega_{\text {conf }}(n+1 / 2)$ and $\chi_{n}(x, y)=\left[\exp \left(i k_{x} x\right) \exp \left(-y^{2} / 2 l^{2}\right) H_{n}(y / l)\right] /\left(\pi^{1 / 2} l L_{x} 2^{n} n !\right)^{1 / 2}$ respectively, where $l=$ $\left(\hbar / m \omega_{\text {conf }}\right)^{1 / 2}$ and $H_{n}(x)$ are the Hermite polynomials. The parameter $l$ gives the scale of electron localization in the $y$ direction ( $l$ yields $3.4 \times 10^{-6} \mathrm{~cm}$ for $\omega_{\text {conf }}=10^{11} \mathrm{~Hz}$ ). Typical values of $\omega_{0}$ are in the range $10^{10}-10^{11} \mathrm{~Hz}$ (for $E_{\perp}=1-3 \mathrm{kV} / \mathrm{cm}$ and $R=5 \times 10^{-4} \mathrm{~cm}$ ). 14] Then the approximation $z_{0} \simeq y^{2} / R$ is rather good and the condition $y \ll R$ is well satisfied.

The electron multisubband spectrum leads to rather interesting transport properties along the channel. The main scattering mechanisms are the electron interaction with atoms in the vapor phase predominating at $T>1 \mathrm{~K}$, the electron-ripplon interaction at lower temperatures, and the electron scattering by surface defects at the helium-substrate interface $(z=-d)$. As it was shown the latter scattering can dominate the SE mobility over a helium 
film in the low temperature regime. [15] The electron mobility in the Q1D channel over bulk helium was calculated in Ref. [14] taking into account the population of the excited subbands in the $y$ direction when $\hbar \omega_{\text {conf }} \lesssim T$ (note that with $\hbar \omega_{\text {conf }} \simeq 0.8 \mathrm{~K}$ for $\omega_{\text {conf }} \simeq 10^{11}$ $\mathrm{Hz}$ ). However the contribution of $n>1$ subbands can be discarded for $T \ll \hbar \omega_{\text {conf. Here we }}$ limit ourselves to this regime and neglect quantum statistics effects (Fermi energy is much smaller than $T)$.

The electron mobility along the channel in the limit $\hbar \omega_{\text {conf }} / T \gg 1$ is given as 14

$$
\mu=\frac{2}{\sqrt{\pi}} \frac{e}{m}\left(\frac{\hbar \omega_{\text {conf }}}{T}\right)^{3 / 2} \int_{0}^{\infty} \frac{\sqrt{x} \exp \left(-\hbar \omega_{\text {conf }} x / T\right)}{\left[\nu_{g}(x)+\nu_{r}(x)+\nu_{d}(x)\right]}
$$

where $x=\hbar k_{x}^{2} /\left(2 m \omega_{\text {conf }}\right)$. The collision frequencies $\nu_{g}(x), \nu_{r}(x)$, and $\nu_{d}(x)$ denote the electron collisions with vapor atoms, ripplons, and defects, respectively.

The collision frequency with helium atoms is given by $\nu_{g}(x)=3 \hbar n_{g} \gamma A / 8 m \sqrt{x}$, where $n_{g}$ is the volume concentration of helium atoms and $A \simeq 4.676 \times 10^{-16} \mathrm{~cm}^{-2}$ is the scattering cross-section. Note that the contribution of $\nu_{g}(x)$ becomes negligible for $T<1 \mathrm{~K}$ because $n_{g}$ decays exponentially with $T$. The electron mobility is dominated from contributions of $\nu_{r}(x)$ and $\nu_{d}(x)$ in that temperature range.

In order to calculate $\nu_{r}(x)$, we use the expression [14

$$
\nu_{r}\left(k_{x}\right)=\frac{2 \pi}{\hbar S} \sum_{\mathbf{q}}\left|\left\langle 0\left|\exp \left(i q_{y} y\right)\right| 0\right\rangle\right|^{2}\left|\left\langle 1\left|V_{r \mathbf{q}}(z)\right| 1\right\rangle\right|^{2}\left(2 N_{q}+1\right) \frac{q_{x}}{k_{x}} \delta\left(E_{k_{x}-q_{x}}-E_{k_{x}}\right) .
$$

Here $E_{k_{x}}=\hbar^{2} k_{x}^{2} / 2 m, \mathbf{q}$ is the $2 \mathrm{D}$ wave vector, and $V_{r \mathbf{q}}(z)=\left[\hbar q \tanh (q d) / 2 \rho \omega_{q}\right]^{1 / 2} e E_{\perp}^{*}$ for a thin helium film, $\omega_{q}^{2}=\left[(\alpha / \rho) q^{3}+g^{\prime} q\right] \tanh (q d)$ is ripplon dispersion law with $g^{\prime}=$ $g+3 n_{H e} \beta /\left(\rho d^{4}\right)$ where $\beta$ is the van der Waals constant of the substrate, $n_{H e}$ is volume concentration in liquid helium. [16] Long wavelength ripplons do mainly contribute to the scattering with $N_{q} \simeq 2 T / \hbar \omega_{q}$. Straightforward calculation of Eq. (3) leads to

$$
\nu_{r}(x)=\frac{e^{2}\left(E_{\perp}^{*}\right)^{2} T}{4 \alpha \hbar^{2} \omega_{\text {conf }}} \cdot \frac{\exp \left[4\left(x+x_{c}\right)\right]}{\sqrt{x^{2}+x_{c} x}}\left[1-\operatorname{erf}\left(2 \sqrt{x+x_{c}}\right)\right] .
$$

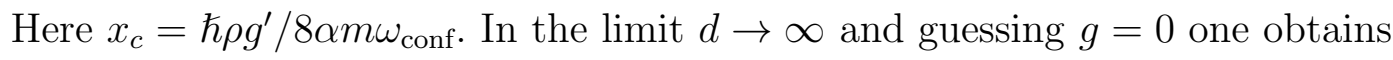

$$
\nu_{r}(x)=\frac{e^{2}\left(E_{\perp}^{*}\right)^{2} T}{4 \alpha \hbar^{2} \omega_{0}} \cdot \frac{\exp (4 x)}{x}[1-\operatorname{erf}(2 \sqrt{x})] .
$$


which reproduces the result of Ref. [11.

The general expression for $\nu_{d}\left(k_{x}\right)$ can be written as

$$
\nu_{d}\left(k_{x}\right)=\frac{2 \pi}{\hbar S} \sum_{\mathbf{q}}\left|\left\langle 0\left|\exp \left(i q_{y} y\right)\right| 0\right\rangle\right|^{2}\left|\left\langle 1\left|V_{d \mathbf{q}}(z)\right| 1\right\rangle\right|^{2}\left|\xi_{s \mathbf{q}}\right|^{2} \frac{q_{x}}{k_{x}} \delta\left(E_{k_{x}-q_{x}}-E_{k_{x}}\right),
$$

where $\xi_{s \mathbf{q}}$ is the Fourier transform of the solid interface displacement from equilibrium position $z=-d, V_{d \mathbf{q}}(z)=-\Lambda_{1} q K_{1}[q(z+d)] /(z+d)$, with $K_{1}(x)$ the modified Bessel function. 15 To calculate the electron-defect contribution, we use the well-known Gaussian two-parameter model for surface defects in which the correlation function $\left\langle\xi(\mathbf{r}) \xi\left(\mathbf{r}^{\prime}\right)\right\rangle=\xi_{0}^{2} \exp \left[-\left|\mathbf{r}-\mathbf{r}^{\prime}\right|^{2} / a^{2}\right]$ depends on $\xi_{0}$ and $a$ playing the roles of characteristic defect height and width respectively. [17] This model leads to $\left\langle\left|\xi_{s q}\right|^{2}\right\rangle=\pi \xi_{0}^{2} a^{2} \exp \left(-q^{2} a^{2} / 4\right)$ and was used to explain the SE transport properties over a thin helium film [15] and over solid hydrogen. [18] The final expression for $\nu_{d}(x)$ is

$$
\nu_{d}(x)=\frac{32 m \Lambda_{1}^{2} \xi_{0}^{2} a^{2} \exp \left(-2 a^{2} x / l^{2}\right)}{\hbar^{3} l^{4} x^{1 / 2}} \Phi_{d}(x)
$$

where

$$
\Phi_{d}(x)=\int_{0}^{\infty} \frac{d y}{\sqrt{y}}(x+y)^{2} \exp \left[-\left(4+\frac{2 a^{2}}{l^{2}}\right) y\right] \varphi_{d}^{2}\left(\frac{\sqrt{2(x+y)}}{\gamma l}\right)
$$

and

$$
\varphi_{d}(x)=\frac{\exp (2 \gamma d)}{x} \int_{2 \gamma d}^{\infty} \frac{d s}{s}(s-2 \gamma d)^{2} K_{1}(x s) \exp (-s)
$$

For a ideal surface substrate $\left(\nu_{d}(x)=0\right)$ the electron mobility is determined by $\nu_{r}(x)$ and is given by

$$
\mu_{r} \simeq 6 \mu_{\perp}\left[1+\frac{32}{3 \pi}\left(\frac{T}{\hbar \omega_{\text {conf }}}\right)^{1 / 2}\right]
$$

for large enough $d$ and $x_{c}$ so small that the condition $T \gg \hbar \omega_{\text {conf }} x_{c}$ is fulfilled, even though $T \ll \hbar \omega_{\text {conf. }}$ Here $\mu_{\perp}=\alpha \hbar /\left[e m\left(E_{\perp}^{*}\right)^{2}\right]$. In case of thin films with $T \ll \hbar \omega_{\text {conf }} x_{c}$, the asymptotic expression for ripplon limited mobility is

$$
\mu_{r} \simeq \frac{8 \mu_{\perp}}{\sqrt{\pi}}\left(\frac{\hbar \omega_{0} x_{c}}{T}\right)^{1 / 2} \frac{\exp \left(-4 x_{c}\right)}{1-\operatorname{erf}\left(2 \sqrt{x_{c}}\right)}
$$


By comparing Eqs. (8) and (9), one can see the drastic change in the temperature dependence of the mobility from a thick to a thin film. One estimates this transition at $d \lesssim 10^{-6}$ $\mathrm{cm}$ for actual substrate materials.

The situation becomes rather interesting when one includes the defect contributions of solid substrates. Indeed for realistic values of $a=10^{-6} \mathrm{~cm}, d$ in the same range, and $\xi_{0}=10^{-7} \mathrm{~cm}, \nu_{d}(x)$ is near two orders of magnitude larger than $\nu_{r}(x)$ for $x=x_{T}=T / \hbar \omega_{\text {conf }}$ and gives the major contribution to the integral of Eq. (2). In such a condition, the defectlimited mobility is given as

$$
\mu_{d} \simeq \frac{e}{m \nu_{d}^{(0)}}\left(\frac{T}{\hbar \omega_{\mathrm{conf}}}\right)^{1 / 2}
$$

valid for above-mentioned values of $\xi_{0}$ and $a$ and $T \gtrsim 0.1 \mathrm{~K}$. Here $\nu_{d}^{(0)}=$ $\pi m \Lambda_{1}^{2} \xi_{0}^{2} a^{2} \gamma^{4} f^{2}(2 \gamma d) / \hbar^{3}$

The results for mobility, given by Eqs. (8-10), are obtained in the single-electron approximation (SEA). Meantime, by increasing electron density, the effects of electron correlations can influence the electron transport properties. To take these effects into account one can apply the so-called complete control approach (CCA) or the Boltzmann shifted distribution approximation, where we assume that the electrons have equal drift velocity $u$ and their distribution function is close to $\exp \left[-\hbar^{2} k_{x}^{2} / 2 m T+\hbar k_{x} u / T\right]$. This approach has been successfully used in calculating the electron mobility in both Q1DES and Q2DES over bulk helium. [14,19] It is valid when the electron-electron collision frequency is significantly larger than the frequencies $\nu_{r}(x), \nu_{g}(x)$, and $\nu_{d}(x)$. The method of calculation is described in details in Ref. [14]. The final expression for the ripplon-limited mobility in the CCA is

$$
\mu_{r}^{(\mathrm{cca})} \simeq 2 \mu_{\perp}\left[1+\frac{4}{\pi}\left(\frac{T}{\hbar \omega_{\mathrm{conf}}}\right)^{1 / 2}\right]
$$

for thick films where $T \gg \hbar \omega_{\text {conf }} x_{c}$. In the opposite limit $\mu_{r}^{(\mathrm{cca})}=(\pi / 4) \mu_{r}$, for $T \ll \hbar \omega_{\text {conf }} x_{c}$ where $\mu_{r}$ is given by Eq. (9). Comparing with Eqs. (8) and (9), one concludes that CCA gives the same qualitatively dependences of the ripplon-limited mobility on temperature and effective holding field as SEA. However the absolute values of mobility are smaller in 
the CCA showing the influence of electron correlations on transport in Q1DES. The defectlimited mobility in CCA is $\mu_{d}^{\text {(cca) }}=\mu_{d} / 4$ where $\mu_{d}$ is given by Eq. (10).

In conclusion we have investigated theoretically the properties of Q1DES over suspended helium films. Film effects modify the confinement potential across the channel and the electron mobility at low temperatures is limited by ripplon scattering and mainly by surface defects at the helium film substrate interface. The latter scattering is dominant for thin films with $d \sim 10^{-6} \mathrm{~cm}$ and leads to the increase of electron mobility with temperature whereas the ripplon-limited mobility should decrease in this limit. Such a prediction can be tested in experimental attempts to observe the influence of different scattering mechanisms in the electron transport of Q1DES over helium film for temperatures below $1 \mathrm{~K}$.

This work was supported by the Fundação de Amparo à Pesquisa do Estado de São Paulo (FAPESP) and the Conselho Nacional de Desenvolvimento Científico e Tecnológico $(\mathrm{CNPq})$. The authors are indebted to Professor F. M. Peeters for discussion of the results. 


\section{REFERENCES}

[1] Yu. Z. Kovdrya and V. A. Nikolaenko, Fiz. Nizk. Temp. 18, 1278 (1998) [Low Temp. Phys. 18, 894 (1992)].

[2] O. I. Kirichek, Yu. P. Monarkha, Yu. Z. Kovdrya, and V. N. Grigor'ev, Fiz. Nizk. Temp. 19, 458 (1993) [Low Temp. Phys. 19, 323 (1993)].

[3] Yu. Z. Kovdrya, V. A. Nikolaenko, H. Yayama, A. Tomokiyo, O. I. Kirichek, and O. B. Berkutov, J. Low Temp. Phys. 110, 191 (1998).

[4] Yu. Z. Kovdrya, V. A. Nikolaenko, S. P. Gladchenko, and S. S. Sokolov, Fiz. Nizk. Temp. 24, 1113 (1998) [Low Temp. Phys. 24, 837 (1998)].

[5] R. J. F. van Haren, G. Acres, P. Fozooni, A. Kristensen, M. J. Lea, P. J. Richardson, A. M. C. Valkering, and R. W. van der Heijden, Physica B 251, 656 (1998).

[6] A. M. C. Valkering and R. W. van der Heijden, Physica B, 251, 652 (1998).

[7] A. M. C. Valkering, Surface electrons in restricted geometry, PhD thesis, Eindhoven (1998), unpublished.

[8] S. P. Gladchenko, V. A. Nikolaenko, Yu. Z. Kovdrya, and S. S. Sokolov, Fiz. Nizk. Temp. 27, 3 (2000) [Low Temp. Phys. 27, 3 (2000)].

[9] J. Klier, I. Doicescu, and Paul Leiderer, J. Low Temp. Phys. 121, 603 (2000).

[10] See for a review, Two-dimensional electron systems in helium and other substrates, edited by E. Y. Andrei (Kluwer, Dordrecht, 1997).

[11] Yu. Z. Kovdrya and Yu. P. Monarkha, Fiz. Nizk. Temp. 12, 1011 (1986) [Low Temp. Phys. 12, 571 (1986)].

[12] A. Valkering, J. Klier, and P. Leiderer, Physica B 284, 172 (2000). See also D. Marty, J. Phys. C: Solid State Phys. 19, 6097 (1986) for earlier attempts. 
[13] S. S. Sokolov, J.-P. Rino, and N. Studart, Phys. Rev. B 55, 14473 (1997).

[14] S. S. Sokolov, G. Q. Hai, and N. Studart, Phys. Rev. B 51, 5977 (1995).

[15] D. Coimbra, S. S. Sokolov, J. P. Rino, and N. Studart, to appear in J. Low Temp. Phys..

[16] Yu. P. Monarkha and V. B. Shikin, Fiz. Nizk. Temp. 8, 563 (1982) [Sov. J. Low Temp. Phys. 8, 279, (1982)].

[17] R. E. Prange and T. W. Nee, Phys. Rev. 168, 779 (1978).

[18] S. S. Sokolov, J.-P. Rino, and N. Studart, Phys. Rev. B 51, 11068 (1995).

[19] V. A. Buntar', V. N. Grigoriev, O. I. Kirichek, Yu. Z. Kovdrya, Yu. P. Monarkha, and S. S. Sokolov, J. Low Temp. Phys. 79, 323 (1990).

\section{FIGURE CAPTION}

Fig. 1. Schematic diagram of a suspended film on a structured substrate. 